\title{
Infrahyoid thyroglossal cyst and Neck trauma - A diagnostic conundrum
}

\author{
PRAMOD CHIRAKKAL ${ }^{1}$, Amira Al Hail ${ }^{1}$, Hala Burzeiza ${ }^{1}$, Ali Al Khafaji ${ }^{1}$, and Shakir Al \\ Mashhadani ${ }^{1}$ \\ ${ }^{1}$ Hamad Medical Corporation
}

February 11, 2021

\begin{abstract}
Thyroglossal duct cysts are most commonly located inferior to the hyoid bone in close relation with the thyrohyoid membrane. Very rarely, they may occupy the posterior hyoid space, and present with dysphagia. We present the clinical, pathophysiologic features and the iintraoperative findings of thisrarely described clinical entity
\end{abstract}

\section{Infrahyoid thyroglossal cyst and Neck trauma - A diagnostic conundrum}

\begin{abstract}
Thyroglossal duct cysts are the most common congenital neck mass. They typically present as a painless midline neck mass in a child or young adult, but may also present later in adulthood when the cyst becomes symptomatic. Thyroglossal duct cysts are most commonly located inferior to the hyoid bone in close relation with the thyrohyoid membrane. Very rarely, they may occupy the posterior hyoid space, and present with dysphagia. We present the clinical, pathophysiologic features and the iintraoperative findings of thisrarely described clinical entity in a young lady.
\end{abstract}

Key words

Thyroglossal cyst, CT Neck, Z- plasty

\section{Case report}

25-year-old female presented to ENT clinic with discharge from the anterior neck and scar(figure1) over the neck for past 10 years .Patient had history of neck trauma in childhood while playing in the park. She was treated by local clinic symptomatically and developed a neck scar without any other symptoms. Patient developed having discharge from the scar site on and off since last 2 years. On detailed clinical examination found to have small sinus tract with discharge while pressure and on CT Neck showed a short sinus tract extending from this scar coursing superiorly in subcutaneous plane, for $2 \mathrm{~cm}$ and ending blindly in close relation to anterior strap muscles(figure $5 \& 6$ ). As per the history, clinical examination and investigation patient diagnosed as post traumatic neck scar with sinus of neck .since patient having a neck scar, we decided to do multidisciplinary approach with plastic surgery of sinus excision and Z-plasty. Simple excision was carried out and intra operatively found tubular structure extending short of hyoid bone around $5 \mathrm{~cm}$ in length(figure 2\&3). Wound closed as Z- plasty technique by plastic surgeon(Figure 4) .Histopathology revealed thyroglossal cyst lined by flattened epithelium surrounded by fibrosis.

\section{Discussion and conclusion}

Thyroglossal duct cyst is the most common congenital midline neck swelling. It results from failure of obliteration of thyroglossal duct which form a bridge between base of tongue and thyroid gland. Thyroglossal 
Duct Cyst usually present as painless cystic mobile fluctuant swelling in close proximity to hyoid bone $[1,2,3]$ which moves with deglutition and protrusion of tongue. In adults one fourth of patients presented with draining sinus that result from spontaneous drainage or surgical drainage of abscess [4].

Thyroglossal Duct Cyst results from failure of involution of thyroglossal duct and present as midline cyst as fistula or sinus in neck which should be properly investigated and diagnosed and should be excised preferably by sistrunk procedure. Our study is small to reach any conclusion but whenever you come across a midline neck swelling,thyroglossal duct cyst should be ruled out. Clinical and radiological findings were altered and limited due to history of neck trauma.This case illustrates the need to consider thyroglossal duct cyst in the differential diagnosis when working up a post-traumatic neck swelling with discharge. A secondary educational objective in this case is to be diligent to consider and rule out laryngeal fracture in the case of a neck mass presenting after trauma as they can easily be missed and present with many overlapping symptoms.

\section{References}

1.Narayana Moorthy S, Arcot R. Thyroglossal duct cyst-more than just an embryological remnant. Indian J Surg. 2011;73(1):28-31. doi: 10.1007/s12262-010-0171-8.

2. Tarcoveanu E, Niculescu D, Elena CA, Vasilescu, Felicia C, Ferariu D, Madalina P, Dorobat D (2009) Thyroglossal Duct Cysts. J de Chirurgie Iasi 5 Nr. 1 ISSN 1584-9341

3. Mohan PS, Chokshi RA, Moser RL, Razvi SA (2005) Thyroglossal duct cysts: a consideration in adults. Am surg 71(6):508-511.

4. Abuabara A, Baratto Filho F, Fuzza RF (2010) Thyroglossal duct cyst. South Braz Dent J 7(2):244-246

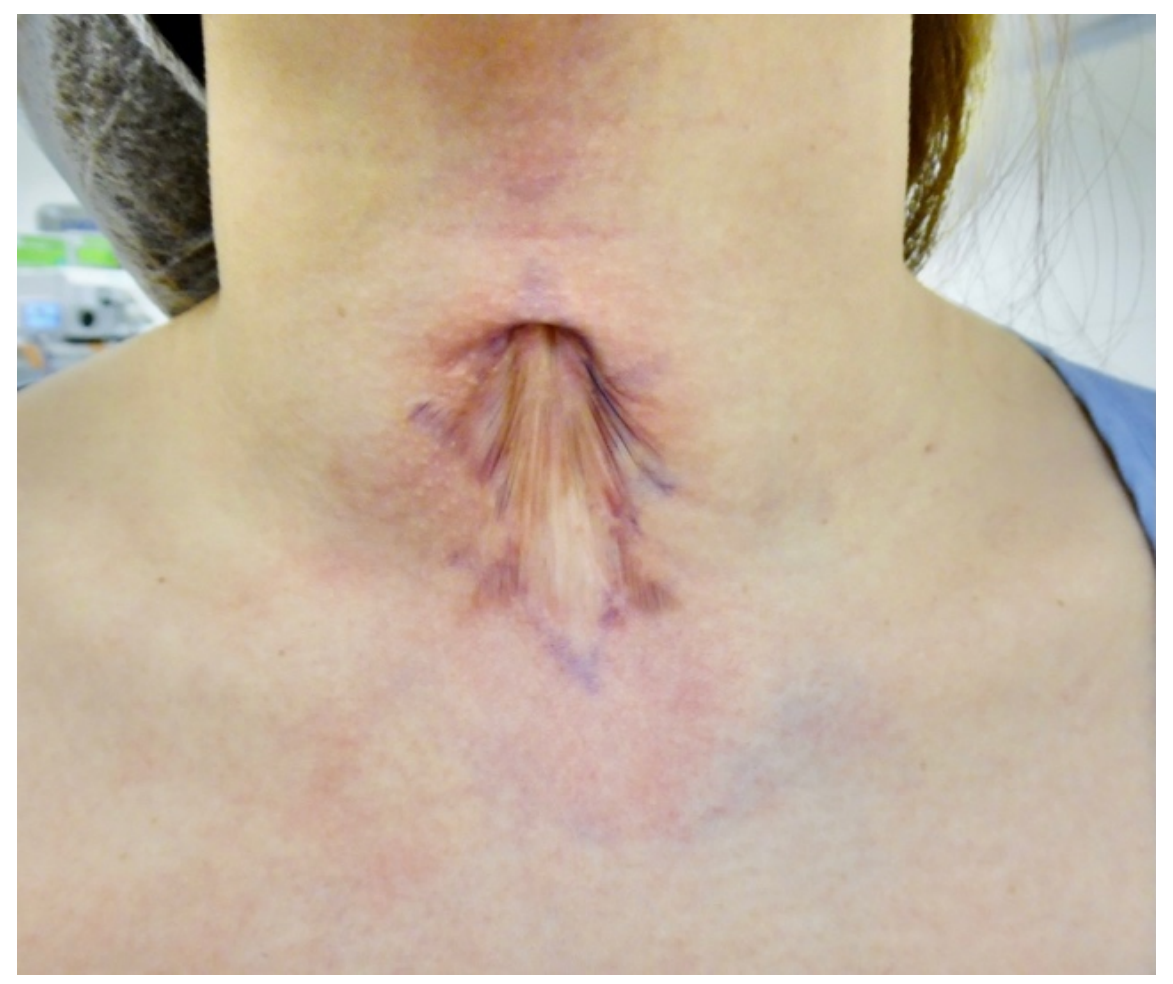

Figure 1 


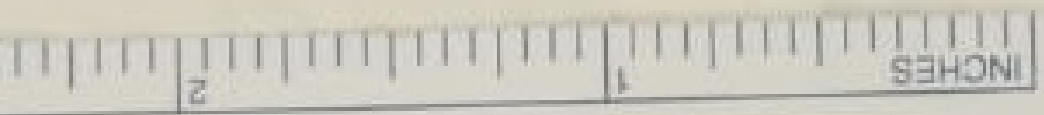
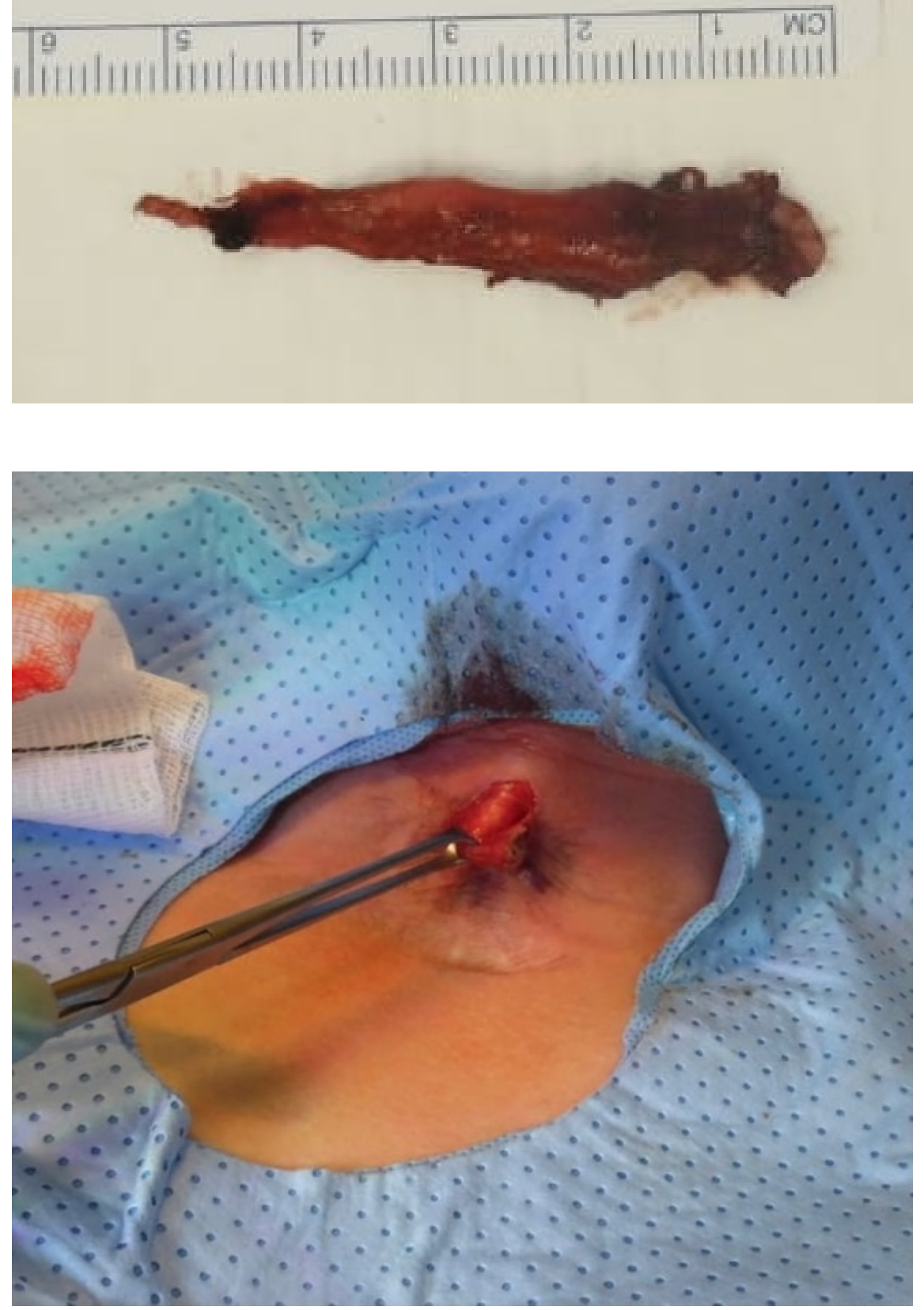

Figure $2 \& 3$ 


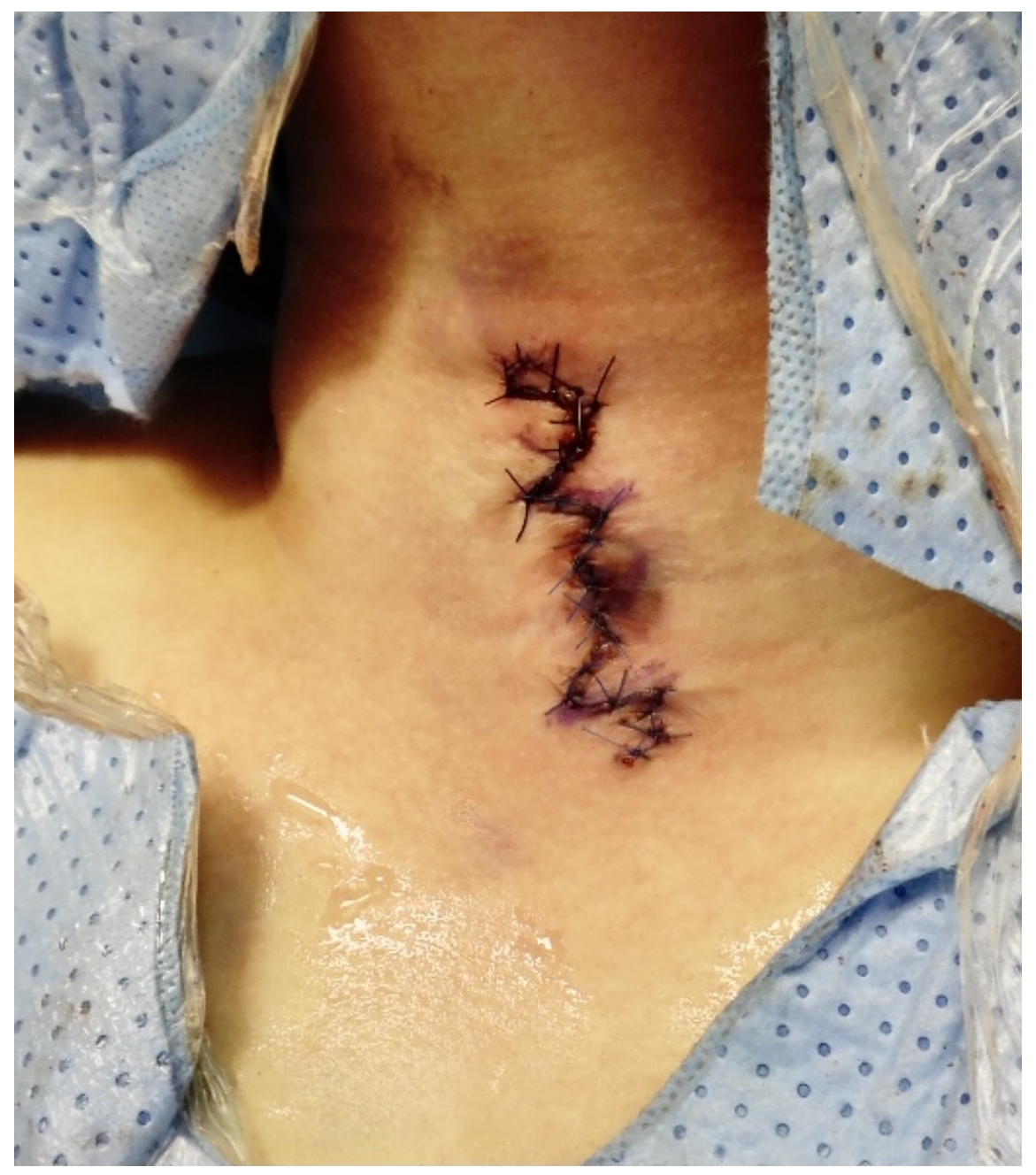

Figure 4 


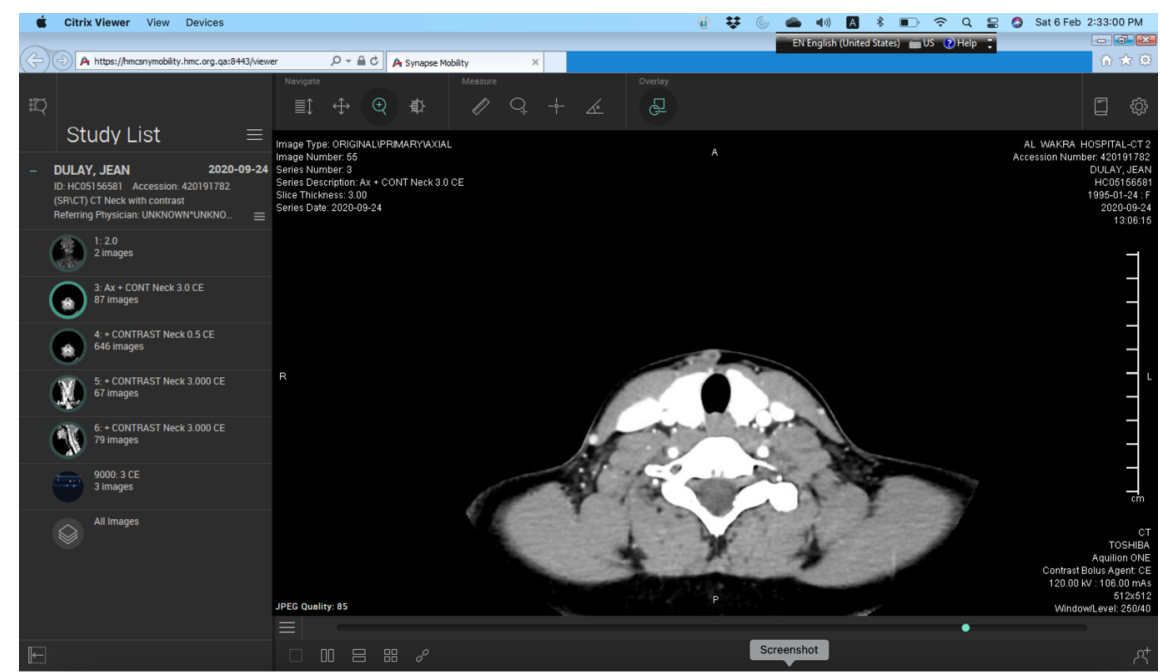

(4)

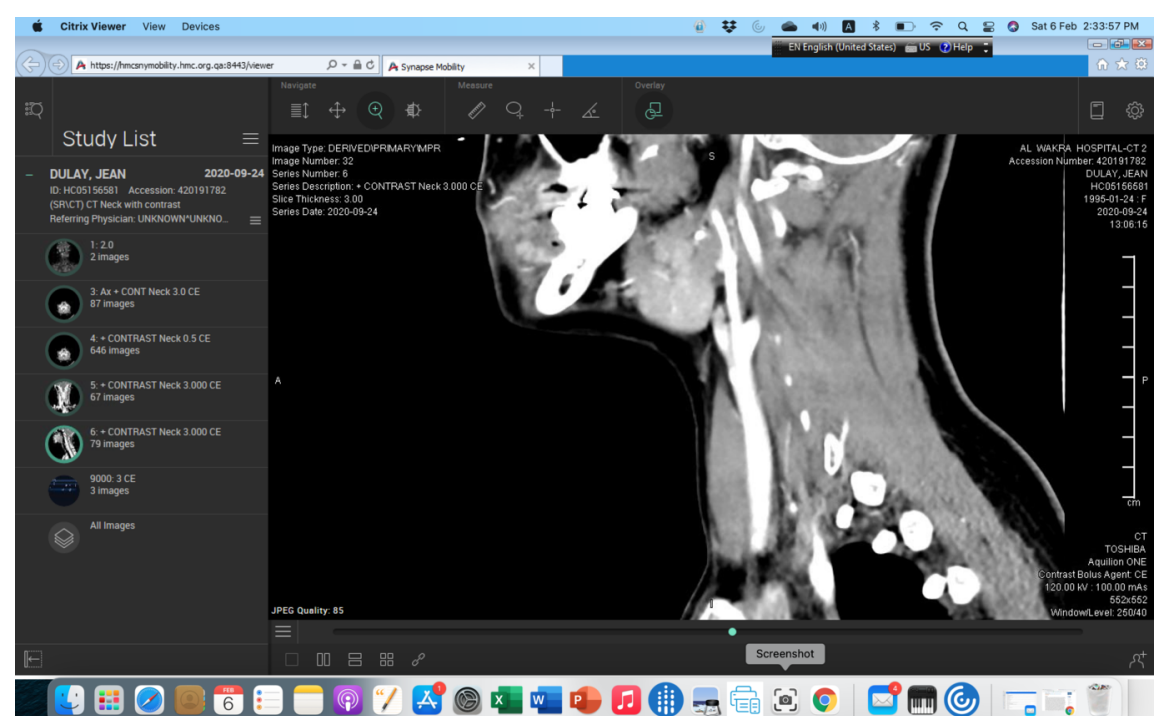

Figure $5 \& 6$

CT Neck with contrast - Focal skin defect representing post-traumatic scar based on the history seen in the anterior part of the neck at the level of thyroid gland, C6 vertebral level. A short sinus tract is seen extending from this scar, coursing superiorly in subcutaneous plane, for $2 \mathrm{~cm}$ and ending blindly in close relation to the anterior strap muscles. No definite internal extension into the larynx or pharynx

\section{Hosted file}

infrahyoid thyroglossal cyst and neck trauma figures.pdf available at https://authorea.com/ users/378948/articles/508505-infrahyoid-thyroglossal-cyst-and-neck-trauma-a-diagnosticconundrum 